Article

\title{
Continuous and Noninvasive Estimation of Right Ventricle Systolic Blood Pressure Using Heart Sound Signal by Deep Bidirectional LSTM Network
}

\author{
Miao Wang, Hong Tang*(D), Tengfei Feng and Binbin Guo \\ School of Biomedical Engineering, Dalian University of Technology, Dalian 116024, China; \\ wangmiao9248@mail.dlut.edu.cn (M.W.); tengfei.feng@mail.dlut.edu.cn (T.F.); \\ guobinbin@mail.dlut.edu.cn (B.G.) \\ * Correspondence: tanghong@dlut.edu.cn
}

Received: 11 July 2020; Accepted: 5 August 2020; Published: 7 August 2020

check for updates

\begin{abstract}
Objective: Timely monitoring right ventricular systolic blood pressure (RVSBP) is helpful in the early detection of pulmonary hypertension $(\mathrm{PH})$. However, it is not easy to monitor RVSBP directly. The objective of this paper is to develop a deep learning technique for RVSBP noninvasive estimation using heart sound (HS) signals supported by (electrocardiography) ECG signals without complex features extraction. Methods: Five beagle dog subjects were used. The medicine U-44069 was injected into the subjects to induce a wide range of RVSBP variation. The blood pressure in right ventricle, ECG of lead I and HS signals were recorded simultaneously. Thirty-two records were collected. The relations between RVSBP and cyclic HS signals were modeled by the Bidirectional Long Short-Term Memory (Bi-LSTM) network. Results: The mean absolute error (MAE) \pm standard deviation (SD) inside record was $1.85 \pm 1.82 \mathrm{mmHg}$. It was $4.37 \pm 2.49 \mathrm{mmHg}$ across record but within subject. The corrective factors were added after training the Bi-LSTM network across subjects. Finally, the MAE \pm SD from $12.46 \pm 6.56 \mathrm{mmHg}$ dropped to $6.37 \pm 4.90 \mathrm{mmHg}$ across subjects. Significance: Our work was the first to apply the Bi-LSTM network to build relations between the HS signal and RVSBP. This work suggested a noninvasive and continuous RVSBP estimation using the HS signal supported by the ECG signal by deep learning architecture without the need of healthcare professionals.
\end{abstract}

Keywords: right ventricular systolic blood pressure; heart sound signal; bidirectional LSTM (Bi-LSTM) network; corrective factors; detection of pulmonary hypertension

\section{Introduction}

Information about the role of the right ventricle (RV) in health and disease historically had fallen behind that of the left ventricle (LV) [1,2]. Comparatively, little attention had been paid on how RV dysfunction may be detected and measured efficiently. RV function may be impaired in pulmonary hypertension $(\mathrm{PH})$, congenital heart disease, and coronary artery disease and in patients with left-sided heart failure or valvular heart disease [3-6]. Previous studies had shown that the potential estimated value of right ventricular blood pressure (RVBP) for patients with right heart failure was needed [7]. Right ventricular systolic blood pressure (RVSBP) is a critical and indispensable indicator for cardiovascular diseases, especially for early discovery and postoperative observation of $\mathrm{PH}$. In the early disease, the symptoms of $\mathrm{PH}$ were often non-specific but progressed over time to functionally limiting dyspnea and fatigue. The non-specific symptoms in the early disease were some of the obstacles to establishing an early diagnosis [8]. Although the natural history varied according to the etiology of the condition, $\mathrm{PH}$ was usually a progressive disease characterized by increased 
pulmonary vascular resistance and diminished RV function due to increased RV afterload. The normal $\mathrm{RV}$ is a thin-walled flow generator able to accommodate large changes in venous return but unable to maintain flow output when a fast increase in pulmonary artery pressure (PAP). Therefore, RVBP increased with the development of PH. In this paper, the authors extracted the RVBP instead of PAP. As far as the author's knowledge, there were no literatures about the prediction of RVBP. The authors compared the prediction of RVSBP with the prediction of PAP.

Right heart catheterization, an invasive procedure, is usually mandatory to confirm the diagnosis of right ventricular disease [9]. However, the financial expense and physical risk of right heart catheterization discourages patients from diagnosis and treatment. Moreover, the right heart catheterization is placed into the heart through a catheter. It is not fit to monitor RVBP or PAP over a long period of time. In clinical practice, there are two possible solutions to diagnose RV diseases non-invasively: Doppler echocardiography and phonocardiography [10]. Doppler echocardiography evaluates the speed of tricuspid regurgitation to calculate PAP [11]. However, some recent studies had suggested that Doppler echocardiographic may frequently be inaccurate [12]. Usually, patients' diagnosis began with a detailed medical history collection and series of physical examinations in hospital. Systematic chest auscultation was crucial for early screening. Heart sound (HS) auscultation was widely used in routine examinations as a noninvasive and low-cost method. In earlier years, researchers found there were relations between the A2-P2 time split interval (TSI), which was further used to estimate PAP based on signal processing of the second heart sounds (S2s) [13-17]. However, the error of the TSI detection affected PAP estimation directly. This limitation prevented the accuracy of PAP estimation. Subsequently, researchers extracted feature sets from the heart sounds and used machine learning algorithms to setup predictive models for PAP estimation [18-20]. Features could be extracted from a wide variety of signal transforms, such as short time Fourier transform (STFT), wavelet transform, S-transform, etc. However, feature extraction was a challenging task. These handpicked features had limitations because they were selected based on a human being's subjective analysis. Dennis and Smith, et al., built the models on human data. The standard deviation (SD) of the best general model were, respectively, $11.7 \mathrm{mmHg}$ and $8.28 \mathrm{mmHg}$ for PAP estimation using heart sounds $[18,19]$. As technology advances, new approaches need to be tried. The problem is still open.

Deep learning had shown strong ability in modelling nonlinear relations from input to output [21]. The authors selected the improved version of recurrent neural network (RNN): Bidirectional Long Short-Term Memory (Bi-LSTM), which was used to model the relations from heart sound signal to RVSBP, automatically. It is known heart sounds are the acoustic vibrations generated by the interaction between heart hemodynamics and heart valves, chamber walls, and great vessels [22,23]. People know little about the complex relations, such as a black box, even though the relations had been noticed for many years. Medical researchers had shown that PAP influenced the characteristics of heart sounds. This suggested that heart sound analysis was a potential method for the noninvasive diagnosis of right ventricular disease [18]. In this paper, the Bi-LSTM network was used to model the black box to link heart sound signal to RVSBP directly. They were validated by experimental data collected from five beagle dog subjects.

\section{Methods}

\subsection{Data Collection}

The experiments were carried out with five healthy adult beagles weighing $10-13 \mathrm{~kg}$. The subjects were anesthetized with pentobarbital sodium $(30 \mathrm{mg} / \mathrm{Kg})$ firstly, then laid down in the supine position for surgical operations. A catheter (Swan-Ganz F7, Edwards, Irvine, CA, USA) filled with a heparinized solution (500 units/mL) was inserted into the RV via the jugular vein. A calibrated catheter and high-fidelity blood pressure transducer (MLTO699, ADInstruments, Sydney, Australia) was used to record the intraventricular blood pressure signal. U-44069 $(40 \mu \mathrm{g} / \mathrm{mL})$ was injected into the upper limb vein of the subjects by a path formed by an intravenous infusion with an uncontrolled flow speed. 
This path remained open during the signal recording so that a new dose of U-44069 could be injected. At the same time, a microphone transducer (MLT201, ADInstruments, Sydney, Australia) was used to record external HS signals at the apex of the heart. The RVBP, HS signal, and (electrocardiography) ECG signal of lead I were simultaneously digitalized at sampling frequency $1 \mathrm{KHz}$ (PL3508, PowerLab 8/35, ADInstruments, Sydney, Australia) with a 16-bit resolution. The microphone, blood pressure transducer, ECG electrical node, and associated electrical lines were kept in fixed positions. The experimental protocol was approved by the ethics committee of the School of Biomedical Engineering, Dalian University of Technology.

The schematic diagram and experimental procedure of the measurement system were shown in Figure 1. The experimental procedure for each subject was divided into three stages. In the first stage, the signals were collected about $60 \mathrm{~s}$ prior to the U-44069 injection. In the second stage, U-44069 was injected into the vein to induce vasoconstriction via activation of the prostaglandin-endoperoxide receptor. The RVBP rose rapidly to a high level. In the third stage, the medicine injection stopped. The RVBP decreased gradually and returned to normal status. This process was repeated 3-10 times for a subject. In total, 32 records were obtained from the five subjects. Every subject was injected with the same dose of the medicine to induce the elevation of right ventricular blood pressure. However, each subject absorbed and metabolized the medicine's ability differently, which lead to the duration of stage 2 and stage 3 to be different. We called this phenomenon the individual difference in this paper. In each trail, the signal analysis was performed prior (stage 1), during (stage 2), and after (stage 3) acute pulmonary artery stenosis. The details of the data were displayed in Table 1, where the 32 trails included two records which only had the normal RVBP (before U44069 injection), such as \#1 and \#25. There were little RVBP changes in the record \#1 and \#25. Other records had a wide of RVBP changes. The minimum of RVSBP was $11.24 \mathrm{mmHg}$ and the maximum was $86.37 \mathrm{mmHg}$. It was shown that the models were evaluated in such wide range of RVSBP. Figure 2 showed an example which gave typical cardiac cycles of the synchronously collected signals. This example was from the \#7 record of Table 1.

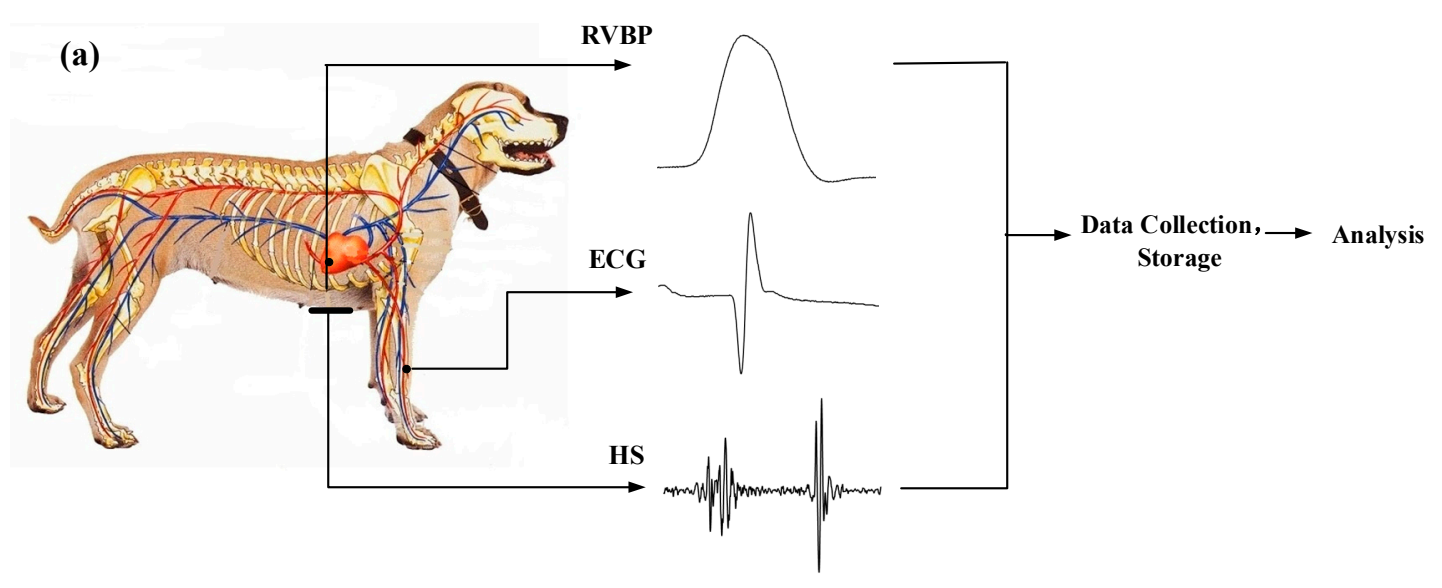

(b)

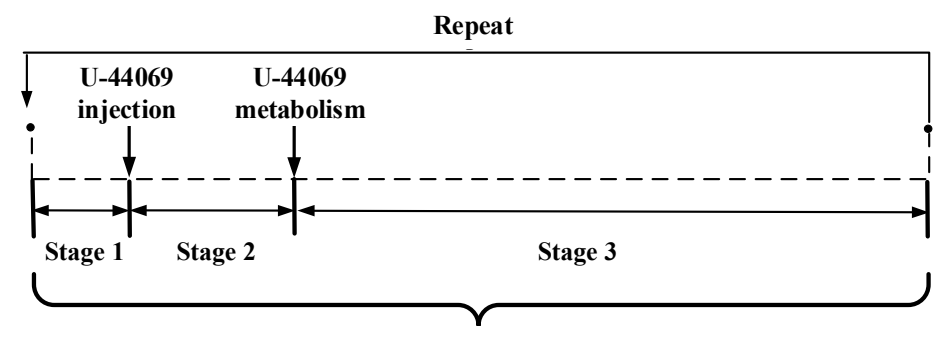

ECG, HS and RVBP signals were collected synchronously in 12-71 minutes.

Figure 1. Schematic diagram of the measurement system and experimental procedure; (a) experiment setup; (b) experimental procedure. ECG: Electrocardiography; HS: Heart Sound; RVBP: Right Ventricular Blood Pressure. 
(a)

(b)

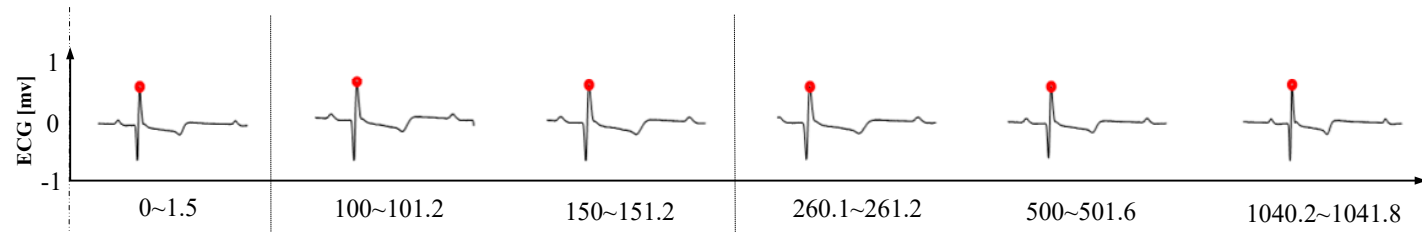

(c)
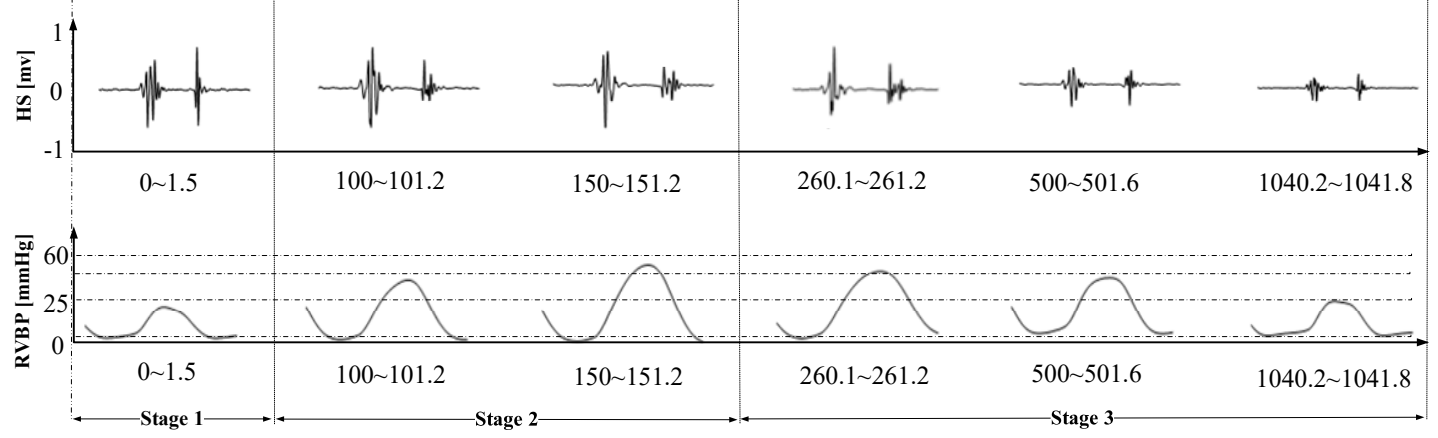

Time in Seconds

Figure 2. An example of synchronously collected signals in a record. Only typical segments were shown here; (a) were the ECG signals; (b) were the heart sound (HS) signals; (c) were the right ventricular blood pressure (RVBP) signals.

Table 1. Summary of the data.

\begin{tabular}{|c|c|c|c|c|}
\hline Subject & No. Record & Time Length (s) & Num. of Cardiac Cycles & RVSBP Range (mmHg) \\
\hline \multirow[t]{11}{*}{ No. 1 (12.5 kg) } & $\# 1$ & 1177.45 & 955 & $23.07-24.54$ \\
\hline & $\# 2$ & 1763.37 & 1193 & $22.38-45.55$ \\
\hline & $\# 3$ & 2202.64 & 1433 & $22.04-48.09$ \\
\hline & $\# 4$ & 1028.04 & 878 & $23.14-57.47$ \\
\hline & $\# 5$ & 910.35 & 861 & $23.68-56.34$ \\
\hline & \#6 & 2214.81 & 1965 & $11.24-50.96$ \\
\hline & \#7 & 1637.72 & 1176 & $21.14-55.73$ \\
\hline & $\# 8$ & 2084.92 & 1301 & $21.46-54.12$ \\
\hline & $\# 9$ & 2202.44 & 1381 & $21.58-53.47$ \\
\hline & $\# 10$ & 1144.53 & 927 & $22.00-54.48$ \\
\hline & $\# 11$ & 1541.69 & 1163 & $21.65-50.65$ \\
\hline \multirow[t]{8}{*}{ No. $2(11 \mathrm{~kg})$} & $\# 12$ & 1128.16 & 914 & $24.80-61.31$ \\
\hline & $\# 13$ & 1844.88 & 1266 & $32.91-56.82$ \\
\hline & $\# 14$ & 2204.93 & 1782 & $29.95-57.06$ \\
\hline & $\# 15$ & 3315.28 & 2271 & $29.81-76.00$ \\
\hline & $\# 16$ & 2698.97 & 2055 & $31.11-68.66$ \\
\hline & $\# 17$ & 4039.60 & 2719 & $22.95-61.70$ \\
\hline & $\# 18$ & 3445.08 & 2307 & $27.56-65.42$ \\
\hline & $\# 19$ & 2282.87 & 2019 & $27.60-53.36$ \\
\hline \multirow[t]{5}{*}{ No. $3(12.5$ kg) } & $\# 20$ & 1180.79 & 951 & $35.59-45.81$ \\
\hline & \#21 & 3457.12 & 2323 & $43.48-75.13$ \\
\hline & $\# 22$ & 3523.08 & 2529 & $32.42-86.37$ \\
\hline & $\# 23$ & 1362.07 & 988 & $41.81-58.19$ \\
\hline & $\# 24$ & 1169.0 & 939 & $42.08-69.02$ \\
\hline \multirow[t]{5}{*}{ No. 4 (10.2 kg) } & $\# 25$ & 524.42 & 449 & $14.57-19.30$ \\
\hline & $\# 26$ & 1508.84 & 1161 & $18.56-44.57$ \\
\hline & \#27 & 1099.17 & 752 & $20.43-44.60$ \\
\hline & $\# 28$ & 520.34 & 355 & $20.83-44.88$ \\
\hline & $\# 29$ & 1432.89 & 1140 & $15.72-48.71$ \\
\hline \multirow[t]{3}{*}{ No. 5 (13 kg) } & $\# 30$ & 914.75 & 646 & $21.82-47.91$ \\
\hline & \#31 & 1345.00 & 967 & $22.09-49.24$ \\
\hline & $\# 32$ & 841.89 & 515 & $22.60-51.17$ \\
\hline
\end{tabular}




\subsection{Data Preprocessing}

The HS signals were filtered with a zero-delay third order butter-worth bandpass filter in the range of 20-200 Hz to remove noise outside the frequency band of heart sounds. The R-waves of ECG signals were recognized by the Pan Tomkins algorithm [24]. In this paper, the ECG signal was used as a reference for HS signal segmentation. It was essential for this research. Heart sounds recording with ECG provide insights into the electrical-mechanical activity of the heart in an unsupervised, non-invasive and inexpensive manner. The first heart sound (S1) occurs immediately after the R-peak (ventricular depolarization) of the ECG. The interval between the current R-peak and next R-peak was called the cardiac cycle. The R-wave of each cardiac cycle indicated the fiducial time of each cardiac cycle of the simultaneous HS signal. The heart sound signals were segmented into consecutive cardiac cycles based on the R-wave positions. Each cardiac cycle of ECG signal corresponded the HS signal which included S1 and the second heart sound (S2), sometimes included the third heart sounds (S3) and the fourth heart sound (S4). It normalized the heart sound signals of all cycles to the same time-scaling of one second. The RVSBP values were detected by the maximum of the RVBP signal in each cardiac cycle. The stretched cyclic heart sound signals were used as inputs to the model and the corresponding RVSBP values were considered as the outputs. The process was shown in Figure 3. Outliers detection and replacement for RVSBP values were sometimes needed because of the motion artifacts or man-made interference [25].

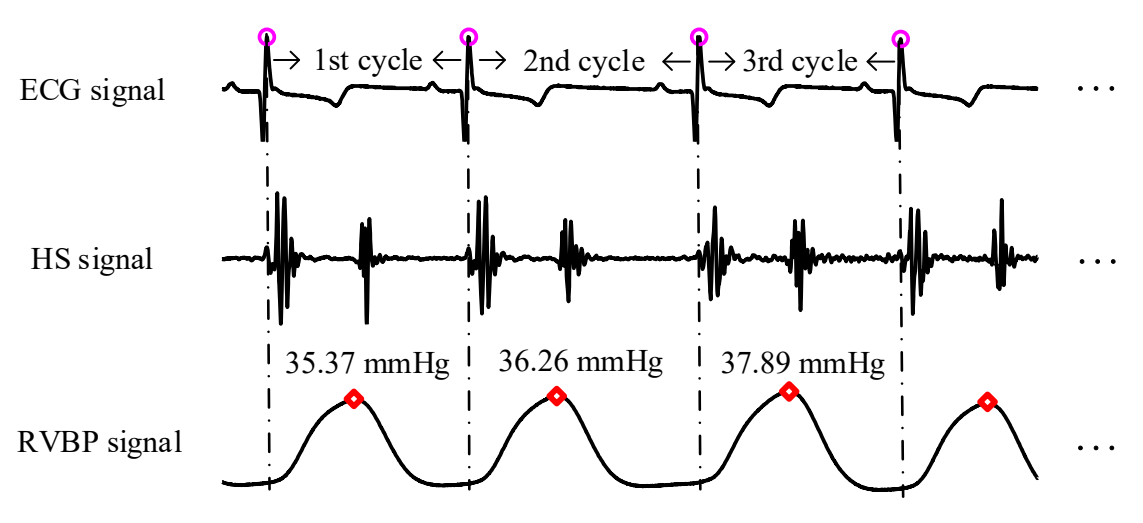

(a)

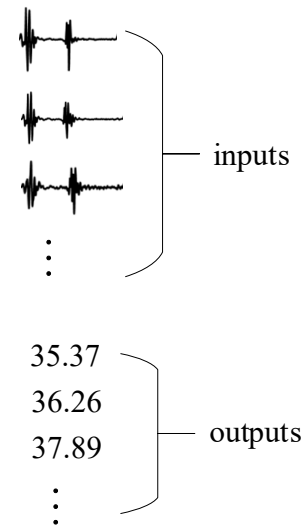

(b)

Figure 3. Constructed the inputs and outputs of the model; (a) segmentation of heart sound signal based on the R-wave positions. The pink circles indicated R-waves. The red diamonds indicated right ventricular systolic blood pressure (RVSBP) values; (b) inputs and outputs to the model.

\subsection{Establishing the Computation Model}

The Long Short-Term Memory (LSTM) units were first proposed by Hochreiter S et al. [26], based on recurrent neural networks. The gating mechanism was used to solve the problem of gradient vanishing. Subsequently, due to their good capability for sequence memory, LSTM units were widely used in the field of natural language processing. An LSTM maintains a memory based on history information, which enables the model to predict the current output conditioned on long distance features. Figure 4 illustrated a single LSTM memory cell. The LSTM memory cell was implemented as the following. The LSTM cell, mainly consisted of the forget gate, input gate, and output gate. Via these gate functions, the LSTM network can effectively capture complicated features within time series in both short and long terms.

$$
\begin{gathered}
i_{t}=\sigma\left(W_{x i} x_{t}+W_{h i} h_{t-1}+W_{c i} c_{t-1}+b_{i}\right) \\
f_{t}=\sigma\left(W_{x f} x_{t}+W_{h f} h_{t-1}+W_{c f} c_{t-1}+b_{f}\right)
\end{gathered}
$$




$$
\begin{gathered}
c_{t}=f_{t} c_{t-1}+i_{t} \tanh \left(W_{x c} x_{t}+W_{h c} h_{t-1}+b_{c}\right) \\
o_{t}=\sigma\left(W_{x o} x_{t}+W_{h o} h_{t-1}+W_{c o} c_{t-1}+b_{o}\right) \\
h_{t}=o_{t} \tanh \left(c_{t}\right) \\
\sigma(x)=\frac{1}{1+e^{-x}} \\
\tanh (x)=\frac{e^{x}-e^{-x}}{e^{x}+e^{-x}}
\end{gathered}
$$

where $\sigma$ is the logistic sigmoid function, and $i, f, o$ and $c$ are the input gate, forget gate, output gate and cell vectors, all of which are the same size as the hidden vector. The weight matrix subscripts have the meaning as the name suggests. For example, $W_{h i}$ is the hidden-input gate matrix, $W_{x o}$ is the input-output gate matrix. The weight matrices from the cell to gate vectors (e.g., $W_{c i}$ ) are diagonal.

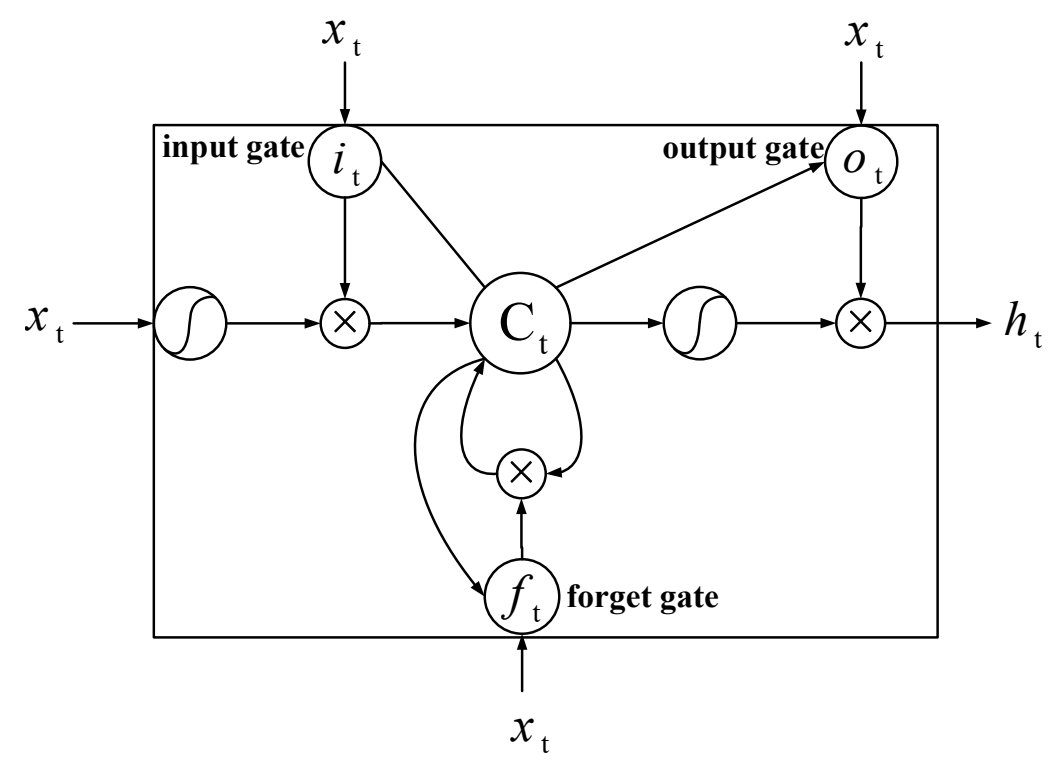

Figure 4. A Long Short-Term Memory Cell.

In this paper, we applied Bi-LSTM [27] to sequence prediction. Bi-LSTM networks were based on the basic LSTM units. The Bi-LSTM model includes forward and backward LSTM channels, which simultaneously captured past and future information. It can extract deep and robust features regarding prediction tasks of time series. The bidirectional LSTM network was showed as Figure 5. The input layer represents input signals and the output layer represents output tags. The inputs and outputs corresponded to the HS signals and the RVSBP values, respectively, as shown in the purple dash line. The purple gradient boxes with rounded corners indicated the LSTM memory cells. The information was processed in both directions with two different hidden layers and fed forward to an output layer. We can efficiently make use of the past inputs (via forward states) and the future inputs (via backward states) for a specific time frame. 


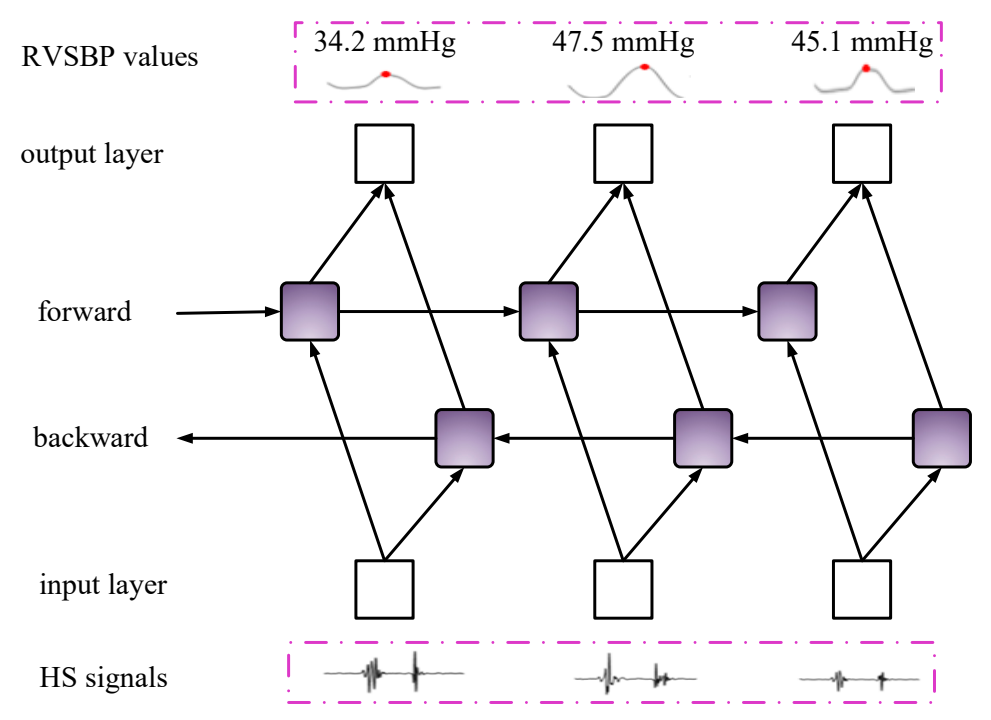

Figure 5. A Bidirectional Long Short-Term Memory (BI-LSTM) network.

Figure 6 was the proposed signal processing framework by the authors. This model was based on supervised learning. The heart sound recordings were segmented into consecutive cardiac cycles based on the R-wave peak locations; at the same time the HS signals were unified into the length of one second time-scaled. In the training stage, the Bi-LSTM model was trained to construct the relations from the inputs (the cyclic heart sound signals) to the outputs (RVSBP values). In the testing stage, the trained model was to estimate RVSBP values with new inputs. Figure 6 illustrated a RVSBP estimation system in which each RVSBP value was tagged with different measured values. For example, in the training set, the Figure 6 showed the HS signals were tagged as $24.2 \mathrm{mmHg}, 58.4 \mathrm{mmHg}, \ldots, 24.7 \mathrm{mmHg}$. In the testing set, the new input HS signals were tagged as the estimated results: $34.2 \mathrm{mmHg}, 47.5 \mathrm{mmHg}, \ldots$, $45 \mathrm{mmHg}$. The input layers had the same dimension with the HS signal size. The output layers had the same dimension with the size of RVSBP. It meant when the data was inputted into the model, the size of the HS signals were $1000 \times N$, where $N$ meant the number of cardiac cycles in each HS record and 1000 meant the uniform length of each HS signal. For the output layers, it also must have the same dimension with the size of the RVSBP. In this paper, the size of RVSBP was $1 \times N$, where $N$ meant the number of cardiac cycles in each RVSBP record, and 1 meant the signal was one dimension.

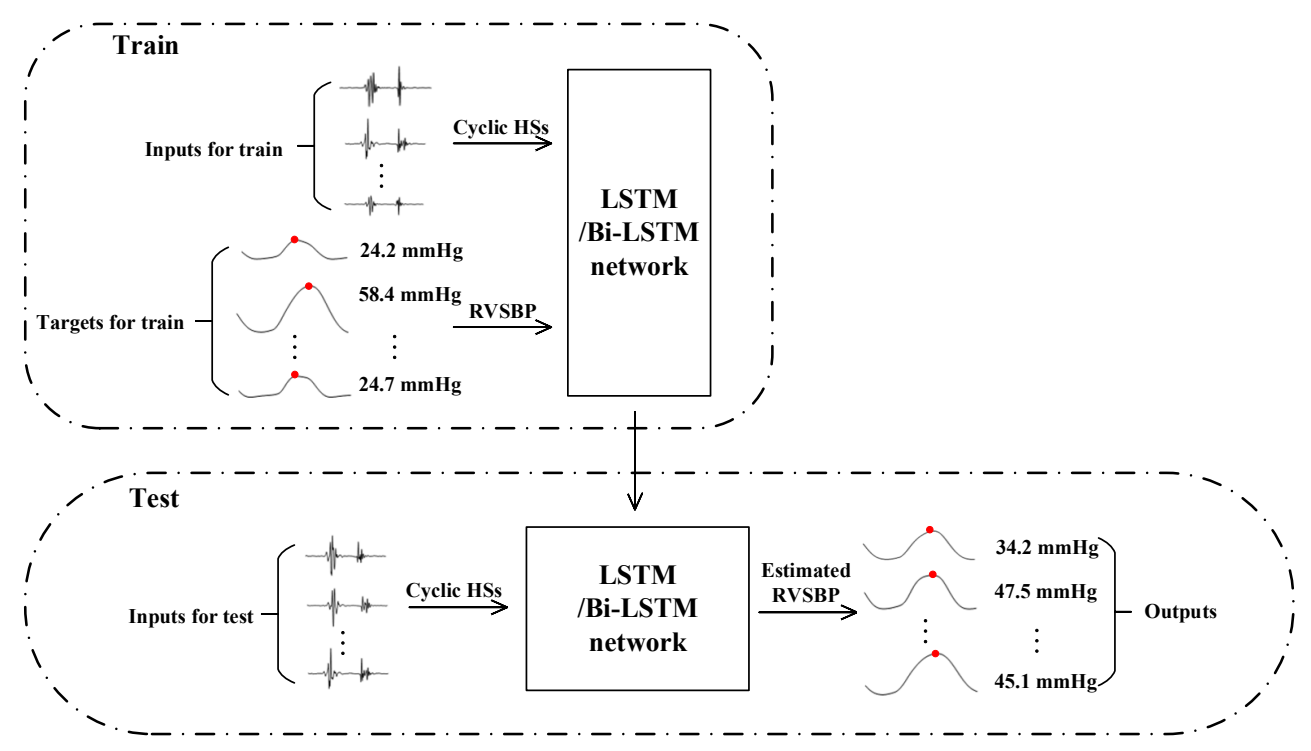

Figure 6. Framework of the proposed technique. 


\subsection{Training Procedure}

In this study, the model contained two bidirectional LSTMs and was briefly called TBLSTM. The TBLSTM networks can automatically select features similar to convolutional neural networks. The TBLSTM model was comprehensively evaluated by three schemes to validate the performance of the proposed technique. The three schemes are defined in the following.

\subsubsection{Evaluation Scheme I: Cross Validation Inside Record}

Scheme I was designed to evaluate the generalization ability of the model inside the record. We evaluated the prediction performance using a five-fold cross validation. A five-fold cross validation was performed by randomly dividing the dataset into equal-sized five partitions. A single partition was used as the validation data for testing the model, and the remaining four partitions were used as training data. This process was repeated five times. Each partition was used once as the validation data. The advantage of five-fold cross validation was that all observations were used for training and testing, and each observation was used for validation once. Parameters were set for the TBLSTM model as follows: the two Bi-LSTM hidden layers both had 80 neurons. The max Epochs was 128, the min Batch Size was 64 and the initial learn rate was 0.01. Cross Entropy was employed to compute the loss function and stochastic gradient descent algorithm was imported to minimize the loss cost in training. The parameters of this group were the best choose based on computer simulations to the authors' knowledge.

\subsubsection{Evaluation Scheme II: Cross Validation Across Records but within Subject}

Scheme II was designed to evaluate the generalization ability across records but within a subject. For example, eleven records were collected from No. 1 subject. The model was trained by ten of these records, and the other was used to test the model. We repeated the process eleven times until each in the subject had experienced a test. For the other subjects, the process was the same as the No.1 subject. Parameters setting for the TBLSTM model were same as those in scheme I.

\subsubsection{Evaluation Scheme III: Cross Validation Across Subjects}

Scheme III was designed to evaluate the generalization ability across subjects. That was, separate the subjects into two non-overlapping groups. One group contained four subjects. All the records of this group were used to train the model. The other group contained the other subject. This subject was used to test the model. We repeated the group separation until each subject experienced testing. The parameters set for the TBLSTM model were the same as those in the Scheme I. In addition, in order to solve the problem of individual differences among different subjects, after the TBLSTM model was trained, corrective factors between individuals were proposed. Firstly, the measured RVSBP subtract the estimated RVSBP, then each subject's errors were recorded. We used one group's features as corrective factors. The group contained the first heart sound's maximum amplitude of each cardiac cycle (S1_max) and the second heart sound's maximum amplitude of each cardiac cycle (S2_max). Features functions were obtained by fitting every subject's errors with the binary linear function. The fitting formulas were as follows:

$$
\begin{aligned}
& \text { errors }=a \cdot S 1 \_m a x+b \cdot S 2 \_m a x+c \\
& R V S B P=\text { estimate_RVSBP }+ \text { errors }
\end{aligned}
$$

where $a, b, c$ represented features combinations' coefficient factors. Coefficients were fitted automaticly. estimate_RVSBP was the estimated result by the TBLSTM model in the scheme III. Finally, the predicted results were the TBLSTM model's predicted outcomes (estimate_RVSBP) added the fitting outcomes (errors) in Scheme III. 


\subsection{Performance Indicators}

Three indicators were defined to evaluate the performance of the models in the three schemes, which were the mean absolute error (MAE) and the standard deviation (SD).

$$
\begin{gathered}
M A E=\frac{1}{N} \sum_{i=1}^{N}\left|P_{i}-P_{i}^{*}\right| \\
M E=\frac{1}{N} \sum_{i=1}^{N}\left(P_{i}-P_{i}^{*}\right) \\
S D=\sqrt{\frac{1}{N-1} \sum_{i=1}^{N}\left(P_{i}-P_{i}^{*}-M E\right)^{2}}
\end{gathered}
$$

where $P$ was the estimated $R V S B P$ value, $P^{*}$ was the measured $R V S B P$ value, and $N$ was the total number of the cardiac cycles in consideration.

\section{Results and Discussions}

The experiment was studied based on five subjects. There were 32 trials in all. The three schemes' results were as follows:

\subsection{Outcomes of Scheme I}

In scheme I, a five-fold cross validation method was used in each record. So, there were five outcomes in every record in the scheme I. To facilitate marking, we used indices to mark the five parts. Table 2 showed the outcomes of the fivefold cross validation. When indices was 1 , the mean MAE \pm SD equaled $1.79 \pm 1.73 \mathrm{mmHg}$; when indices was 2 , the mean MAE \pm SD equaled $1.79 \pm 1.76 \mathrm{mmHg}$; when indices was 3 , the mean $\mathrm{MAE} \pm \mathrm{SD}$ equaled $1.91 \pm 1.84 \mathrm{mmHg}$; when indices was 4 , the mean $\mathrm{MAE} \pm \mathrm{SD}$ equaled $1.81 \pm 1.79 \mathrm{mmHg}$; whenindices was 5 , the mean $\mathrm{MAE} \pm \mathrm{SD}$ equaled $1.99 \pm 2.00 \mathrm{mmHg}$. The scheme I's mean MAE $\pm \mathrm{SD}$ was $1.85 \pm 1.82 \mathrm{mmHg}$. In the scheme I both MAE and SD were low.

\subsection{Outcomes of Scheme II}

In scheme II, the model was evaluated within subject. The TBLSTM model's performance of the five subjects was shown in Figure 7. Where the blue solid curve was the measured RVSBP values, and the red solid curve was the estimated RVSBP values. It was seen that the estimated RVSBP followed the measured RVSBP very well. The MAEs and the SDs were also listed in Table 2 for further analysis. The outcomes showed that although the features were not extracted by hand, we used the Bi-LSTM model which can track changes of the RVSBP with low MAE and SD. Hence, RVSBP can be accurately predicted even when not using the HS features. 

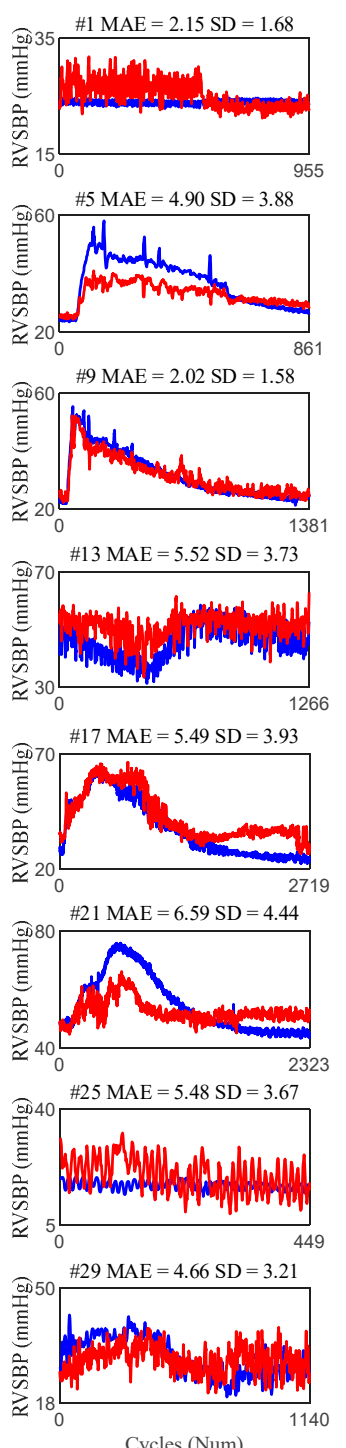

Cycles (Num)
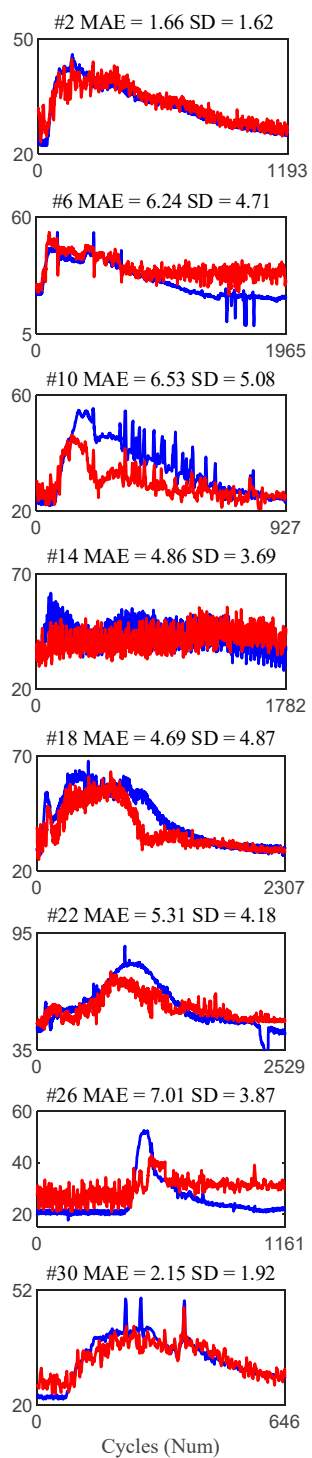
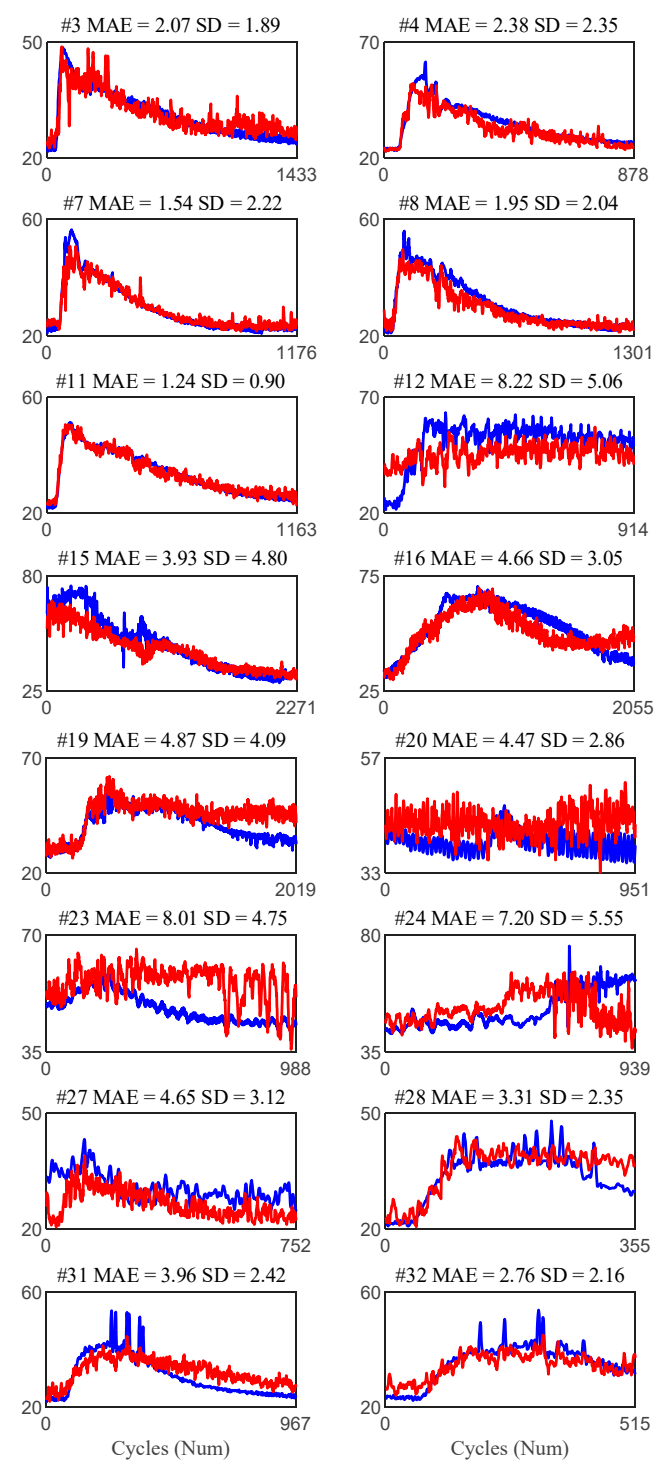

Figure 7. Performance of scheme II.

\subsection{Outcomes of Scheme III}

We had validated that the performances of the scheme I and scheme II were good. Both of the MAE and SD inside trials and across trials were low. The objective of the scheme III was to evaluate the TBLSTM model's generalization ability among different subjects. The MAE and SD were given in column "Scheme III Bi-LSTM" in Table 2. The "corrections" way showed corrective factors were added after training the TBLSTM model. The final results of the scheme III were calculated as the formula in (8)-(9). The results of the "corrections" way was showed in column "scheme III corrections" in Table 2. Figure 8 was the five subjects' estimated result. The blue solid curve was the measured RVSBP. The red solid curve was the TBLSTM model's estimated RVSBP. The pink solid curve was the outcomes of adding the corrective factors. 

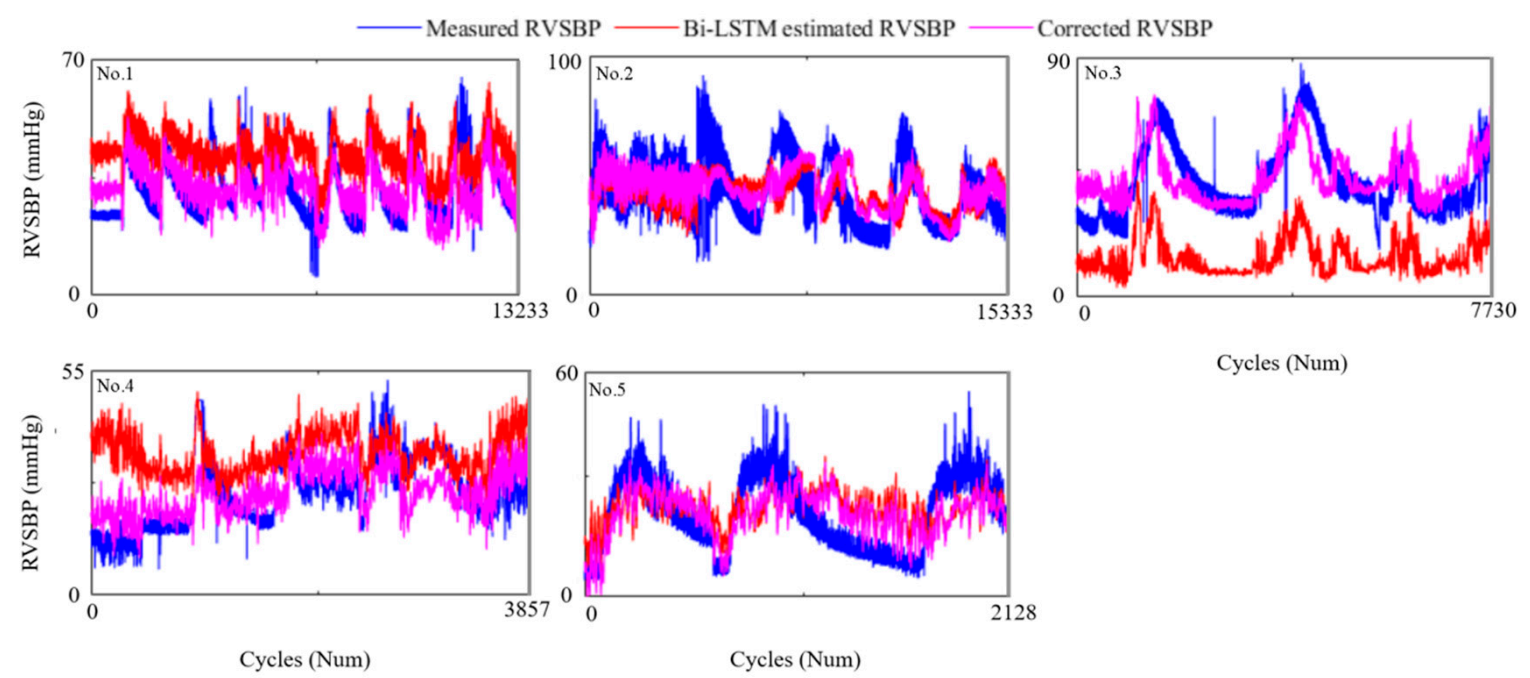

Cycles (Num)

Figure 8. Performance of scheme III.

\subsection{Summary of the Evaluation}

Table 2 showed the MAE and SD of the three schemes. The color coding gave a good overview of the model's performance. The white color meant lower errors than the pink color. The average of MAE and SD were calculated in the last row in Table 2, where there are the results of MAE1 to MAE5 and SD1 to SD5 for scheme I, the results of MAE6 and SD6 for scheme II, the results of MAE7 to MAE8, and SD7 to SD8 for scheme III. The average of MAE and SD were $1.85 \mathrm{mmHg}$ and $1.82 \mathrm{mmHg}$ for scheme I, and $4.37 \mathrm{mmHg}$ and $2.49 \mathrm{mmHg}$ for scheme II. The MAE and SD became a little great in "scheme III Bi-LSTM", because different subjects had individual differences. However, the corrective factors were added after the "scheme III Bi-LSTM". Both of the MAE and SD dropped in "scheme III corrections". The MAE \pm SD from $12.46 \pm 6.56 \mathrm{mmHg}$ downed to $6.37 \pm 4.90 \mathrm{mmHg}$. Figure 9a-c showed the box plots of the three schemes, where the high MAE and SD values were shown by red crosses " + ". We can see the errors were controlled below $3 \mathrm{mmHg}$ in the scheme I, $5 \mathrm{mmHg}$ in the scheme II, and $7 \mathrm{mmHg}$ after adding the corrective factors in the scheme III. Generally speaking, the three schemes showed that the proposed method was a convenient method to estimate RVSBP non-invasively. The model was suitable for estimating RVSBP even in the situation of very high and very low RVSBP values. The method proved that the model had stable generalization ability within trials, across trials, and across subjects, with the addition of correction factors. Overall, continuous RVSBP tracking based on HS signals, no matter short or long sequences, using the proposed method could get good results. 
Table 2. The estimation performances of Bi-LSTM model for three schemes.

\begin{tabular}{|c|c|c|c|c|c|c|c|c|c|c|c|c|c|c|c|c|c|}
\hline \multirow{3}{*}{ Subject } & \multirow{3}{*}{ No. Record } & \multicolumn{10}{|c|}{ Scheme I } & \multirow{2}{*}{\multicolumn{2}{|c|}{$\begin{array}{c}\text { Scheme II } \\
\text { LSTM }\end{array}$}} & \multicolumn{4}{|c|}{ Scheme III } \\
\hline & & \multicolumn{2}{|c|}{ Indices $=1$} & \multicolumn{2}{|c|}{ Indices $=2$} & \multicolumn{2}{|c|}{ Indices $=3$} & \multicolumn{2}{|c|}{ Indices $=4$} & \multicolumn{2}{|c|}{ Indices $=5$} & & & \multicolumn{2}{|c|}{ Bi-LSTM } & \multicolumn{2}{|c|}{ Corrections } \\
\hline & & MAE 1 & SD1 & MAE2 & SD2 & MAE3 & SD3 & MAE4 & SD4 & MAE5 & SD5 & MAE6 & SD6 & MAE7 & SD7 & MAE8 & SD8 \\
\hline \multirow{11}{*}{ No. 1} & $\# 1$ & 0.21 & 0.21 & 0.24 & 0.19 & 0.25 & 0.16 & 0.19 & 0.13 & 0.28 & 0.15 & 2.15 & 1.68 & \multirow{11}{*}{11.77} & \multirow{11}{*}{5.88} & \multirow{11}{*}{5.85} & \multirow{11}{*}{4.83} \\
\hline & $\# 2$ & 1.01 & 1.06 & 0.92 & 0.99 & 0.69 & 0.72 & 0.70 & 0.79 & 1.40 & 1.20 & 1.66 & 1.62 & & & & \\
\hline & \#3 & 0.88 & 1.23 & 1.05 & 0.91 & 0.98 & 1.22 & 1.14 & 0.96 & 0.89 & 1.10 & 2.07 & 1.89 & & & & \\
\hline & $\# 4$ & 1.26 & 1.81 & 1.00 & 1.18 & 1.20 & 1.53 & 0.95 & 1.13 & 1.06 & 1.38 & 2.39 & 2.35 & & & & \\
\hline & $\# 5$ & 1.39 & 1.08 & 1.45 & 2.08 & 2.23 & 2.58 & 1.21 & 1.12 & 1.79 & 2.11 & 4.86 & 3.75 & & & & \\
\hline & \#6 & 1.11 & 1.19 & 1.02 & 1.02 & 1.06 & 1.45 & 1.16 & 1.88 & 1.23 & 1.73 & 6.21 & 4.67 & & & & \\
\hline & \#7 & 1.53 & 1.23 & 1.18 & 1.43 & 1.73 & 1.71 & 1.36 & 1.73 & 1.58 & 1.11 & 1.53 & 2.22 & & & & \\
\hline & $\# 8$ & 1.12 & 1.15 & 1.74 & 1.05 & 2.05 & 1.24 & 1.42 & 1.20 & 1.42 & 1.97 & 1.94 & 2.01 & & & & \\
\hline & \#9 & 1.20 & 1.13 & 1.25 & 1.57 & 1.23 & 1.38 & 1.53 & 2.12 & 1.16 & 1.00 & 2.01 & 1.55 & & & & \\
\hline & $\# 10$ & 2.36 & 1.44 & 1.76 & 2.52 & 1.82 & 1.89 & 1.71 & 2.03 & 1.93 & 1.25 & 6.47 & 5.07 & & & & \\
\hline & $\# 11$ & 1.26 & 1.74 & 0.88 & 0.91 & 1.21 & 1.09 & 1.42 & 1.05 & 0.62 & 0.55 & 1.24 & 0.90 & & & & \\
\hline \multirow[t]{8}{*}{ No. 2} & $\# 12$ & 4.00 & 4.67 & 3.58 & 3.66 & 4.20 & 3.35 & 3.43 & 2.87 & 3.32 & 2.64 & 8.23 & 5.05 & \multirow{8}{*}{9.80} & \multirow{8}{*}{7.37} & \multirow{8}{*}{8.94} & \multirow{8}{*}{6.93} \\
\hline & $\# 13$ & 4.00 & 2.80 & 3.28 & 2.28 & 3.24 & 2.52 & 3.45 & 2.62 & 3.90 & 2.77 & 5.50 & 3.73 & & & & \\
\hline & \#14 & 2.50 & 2.31 & 2.80 & 2.38 & 2.63 & 2.25 & 2.77 & 2.15 & 2.77 & 2.27 & 4.86 & 3.69 & & & & \\
\hline & \#15 & 1.78 & 1.75 & 2.05 & 3.01 & 2.38 & 3.28 & 2.77 & 2.83 & 2.86 & 6.78 & 3.81 & 3.85 & & & & \\
\hline & $\# 16$ & 1.26 & 1.08 & 1.47 & 1.32 & 1.55 & 1.31 & 1.15 & 1.15 & 1.66 & 1.32 & 4.65 & 3.05 & & & & \\
\hline & $\# 17$ & 0.89 & 1.11 & 0.93 & 0.98 & 1.12 & 1.20 & 1.01 & 1.09 & 1.28 & 1.29 & 5.48 & 3.93 & & & & \\
\hline & $\# 18$ & 1.40 & 1.78 & 1.32 & 1.57 & 1.22 & 1.40 & 1.40 & 1.69 & 1.37 & 1.66 & 4.66 & 4.88 & & & & \\
\hline & \#19 & 1.44 & 1.30 & 1.37 & 1.13 & 1.37 & 1.30 & 1.69 & 1.79 & 1.35 & 1.27 & 4.85 & 4.08 & & & & \\
\hline No. 3 & $\# 20$ & 0.80 & 0.87 & 1.67 & 1.58 & 0.84 & 1.13 & 1.01 & 1.64 & 0.71 & 0.55 & 4.47 & 2.86 & & & & \\
\hline & \#21 & 0.61 & 0.62 & 0.72 & 0.68 & 0.79 & 0.57 & 0.98 & 1.08 & 0.81 & 0.84 & 6.59 & 4.44 & & & & \\
\hline & \#22 & 1.15 & 1.40 & 1.23 & 1.25 & 1.16 & 1.61 & 1.07 & 1.20 & 1.31 & 1.52 & 5.30 & 4.16 & 24.95 & 8.97 & 6.39 & 5.22 \\
\hline & $\# 23$ & 0.62 & 0.48 & 0.67 & 0.57 & 0.59 & 0.45 & 0.71 & 0.62 & 0.78 & 0.59 & 8.01 & 4.75 & & & & \\
\hline & $\# 24$ & 1.57 & 1.61 & 1.91 & 1.55 & 2.25 & 3.18 & 1.93 & 2.11 & 2.01 & 2.43 & 7.20 & 5.55 & & & & \\
\hline No. 4 & $\# 25$ & 0.94 & 1.14 & 0.88 & 1.09 & 0.69 & 0.47 & 0.89 & 1.82 & 0.89 & 1.05 & 5.48 & 3.67 & & & & \\
\hline & \#26 & 2.07 & 2.31 & 1.96 & 2.41 & 2.42 & 2.95 & 2.27 & 1.97 & 2.15 & 4.16 & 7.00 & 3.87 & & & & \\
\hline & $\# 27$ & 2.60 & 2.44 & 2.12 & 2.08 & 2.28 & 1.86 & 2.46 & 2.05 & 2.66 & 2.42 & 4.65 & 3.12 & 9.68 & 6.65 & 5.38 & 3.81 \\
\hline & \#28 & 4.84 & 5.06 & 4.50 & 3.58 & 5.56 & 5.04 & 4.79 & 4.41 & 7.02 & 5.39 & 3.25 & 2.35 & & & & \\
\hline & $\# 29$ & 2.56 & 2.01 & 2.92 & 2.36 & 2.82 & 1.98 & 2.68 & 2.38 & 3.18 & 2.09 & 4.66 & 3.21 & & & & \\
\hline No. 5 & $\# 30$ & 3.57 & 2.67 & 3.48 & 2.38 & 2.62 & 2.48 & 2.81 & 2.24 & 2.96 & 3.07 & 2.09 & 1.70 & & & & \\
\hline & \#31 & 2.61 & 2.24 & 3.33 & 4.27 & 2.72 & 1.85 & 2.64 & 2.53 & 2.74 & 3.06 & 3.89 & 2.24 & 6.09 & 3.93 & 5.29 & 3.73 \\
\hline & \#32 & 2.59 & 2.34 & 2.58 & 2.21 & 4.09 & 3.87 & 3.08 & 2.73 & 4.55 & 3.26 & 2.69 & 2.00 & & & & \\
\hline & mean & 1.79 & 1.73 & 1.79 & 1.76 & 1.91 & 1.84 & 1.81 & 1.79 & 1.99 & 2.00 & 4.37 & 2.94 & 12.46 & 6.56 & 6.37 & 4.90 \\
\hline
\end{tabular}

Note: The depth of the color represented the degree of the error. The white color meant lower errors than the pink color. 

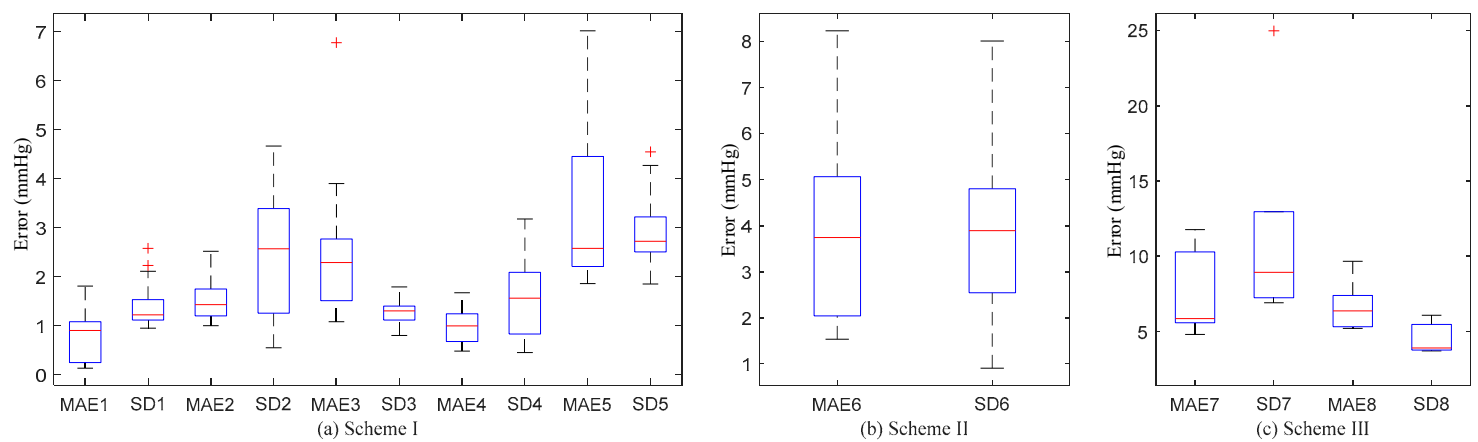

Figure 9. Box plots of the model performance. (a) MAE1-MAE5 and SD1-SD5 of scheme I, (b) MAE6 and SD6 of scheme II, (c) MAE7-MAE9 and SD7-SD9 of scheme III.

\subsection{Discussions}

Previous studies had shown that PAP can be estimated by heart sound features [28-33]. Stephen [28] proposed to use neural network to estimate PAP in 1999 firstly and got standard estimated error $1.9 \mathrm{mmHg}$. The error was low. However, it was better to use cross-validation to test their model. After that, Smith R [19] extracted 38 features on different domains and used the feature selection method to select good features. They used cross validation methods and got a mean error of $8.3 \mathrm{mmHg}$, which may be the best performance at that time, to the authors' knowledge. Researchers [29-33] usually analyzed the relations between HS signals' time domain, frequency domain, amplitude domain, entropy domain features, and PAP. Then, they used machine learning to estimate the PAP. However, their focuses were on the feature's analysis. At the same time, the left ventricular blood pressure (LVBP) and aortic pressure were also researched by the investigators [34-37]. The way to predict LVBP or aortic pressure was similar with the process of PAP prediction. They always separated the first and second heart sounds, known as S1 and S2, from the directly measured heart sound signals. However, precise automated localization of the S1 and S2 or measurement of the splitting interval between the components of the S2 had proved difficult. In summary, it could be found that the estimated performance was related to the extracted features directly. However, feature extraction or feature selection was still a big problem.

This paper suggested a deep learning based on Bi-LSTM to estimate RVSBP using heart sound signals without complex feature extraction. Bi-LSTM is an indirection ally connected network with two layers of LSTM, it gives full consideration to the relationship between the current data and the data before and after it. Bi-LSTM solves the gradient disappearance or gradient explosion problem in RNN, and overcomes the drawback that LSTM only considers the relationship between the current data and its previous data. In this paper, we chose Bi-LSTM rather than LSTM; we thought that the prediction of the time series not only needs to consider the previous data but also the later data. So, we chose the Bi-LSTM. To illustrate the performance of the Bi-LSTM network, we performed multiple sets of experiments in the RVSBP estimation based on the HS signal. It was comprehensively evaluated by three schemes, i.e., inside trial (scheme I), across trials but within subject (scheme II), and across subjects (scheme III). Both scheme I and scheme II could get good estimated results. If we trained and tested the data between individuals directly, the effects were not good (such as "scheme III Bi-LSTM"), because of the individual differences between subjects. Individual difference means that when the basic conditions are the same, there will be significantly different reactions between individuals, such as high sensitivity reaction, low sensitivity reaction, and specific reaction. In this paper, the reasons for individual differences were extensive and complicated, mainly due to the absorption and metabolism of drugs being different for different subjects. The blood concentration of the same dose of drugs in a different individual is different, so that the intensity and duration of the drug action are very different for different individuals. In this paper, each individual was injected with the same dosage of the drug by the experiment operator. However, the subjects had different absorptions and metabolisms. 
In Figure 10, the histograms of RVSBP distribution between individuals were shown to compare the RVSBP differences across subjects. The dark blue histogram represented RVSBP distribution. The red curve represented the fitted Gaussian distribution curve based on the RVSBP distribution histogram. The fitting parameters $(\mu$ and $\sigma$ ) were obtained. The Figure 10 showed the RVSBP value distributions of No.1, No.4, and No.5 were roughly from 20 to $50 \mathrm{mmHg}$. However, the rang of RVSBP value distributions of No.2 and No.3 were larger than the range of No.1, No.4, and No.5. Due to the individual differences, the authors added the corrective factors to correct errors between individuals. Finally, the estimations of RVSBP between different individuals got better estimated outcomes than before in scheme III. As shown in Figure 8, although the absolute value between the measured RVSBP and the estimated RVSBP differed, the relative value difference was small. The trend between the estimated RVSBP and the real RVSBP was very close. Such as No.1, No.3, No.4, and No.5 in Figure 8, the trends of estimated RVSBP were basically consistent with the trends of the measured RVSBP. Table 3 showed the regression coefficient. Combined the distribution of the RVSBP data and the regression coefficient distribution of the feature's analysis, we found that the higher of the distribution of RVSBP values, the higher of the weight of the S1_max. This finding maybe used as a trend to predict RVSBP. In our work, the Bi-LSTM network did not require complex feature extraction techniques and we could estimate the trend of RVSBP.
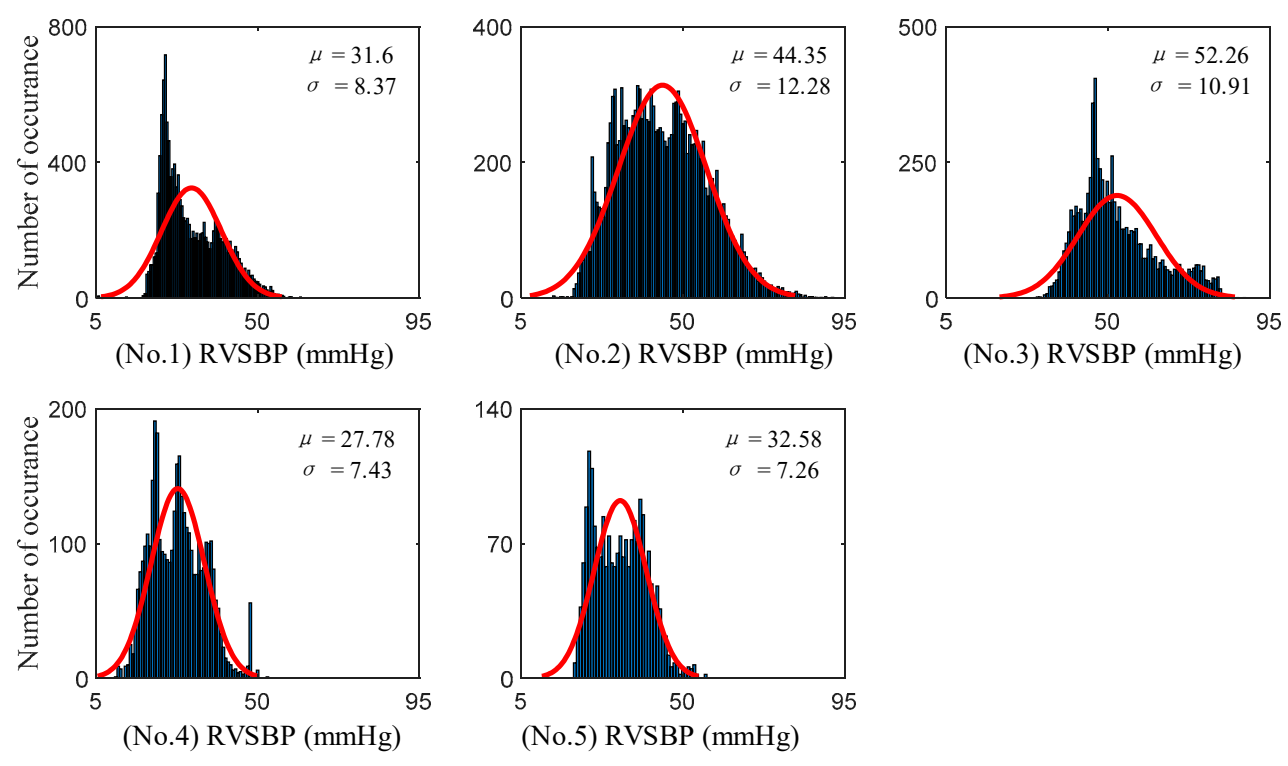

Figure 10. Histogram of RVSBP distribution.

Table 3. Summary of regression coefficients.

\begin{tabular}{cccc}
\hline \multirow{2}{*}{ Subject } & \multicolumn{3}{c}{ Coefficient: $\mathbf{a}, \mathbf{b}, \mathbf{c}$} \\
& $\mathbf{a}$ & $\mathbf{b}$ & $\mathbf{c}$ \\
\hline 1 & 16.49 & -0.29 & -11.52 \\
2 & 30.42 & -0.70 & -4.36 \\
3 & 35.74 & -1.72 & 26.63 \\
4 & -3.10 & -0.95 & -1.15 \\
5 & -32.60 & 1.16 & -3.03 \\
\hline
\end{tabular}

Although our method could estimate the RVSBP non-invasively, we also discovered drawbacks from the wrong estimated samples. As shown in scheme II \#20,\#23, and \#24 from Figure 7, the three records were from the subject 3 . At the same time, we could see the outcome of the subject 3 in the scheme III which outcome was the worst in five subjects. It is probably due to this sample's range of RVSBP being large, so that the individual difference of the subject 3 was bigger than other subjects. Eventually, the outcome of the estimation of the subject 3 was worse than others. This problem may 
be solved in two ways. On the one hand, the authors found the trends of the predicted results were good, but there was a constant between the measured RVSBP and the predicted RVSBP. If we could find the pathological mechanism or the relationship between the HS features and the RVSBP. Maybe the predicted results could compensate a constant and then the predicted results will be accurate. On the other hand, we need to add the number of the subjects. So that there were enough subjects, the model could learn and validate the relation fully. We need to reduce our gap between the different individuals and elevate the accuracy of the RVSBP forecasts in the future study. Besides, the RVSBP was influenced by breathing. So, the signal was slightly up and down, fluctuating during the process of collecting. Eventually the collected RVSBP signal was not smooth. In the next step, we need to try to solve these problems.

\section{Conclusions}

The authors had proposed the Bi-LSTM model for continuous and noninvasive RVSBP estimation using HS signals supported by ECG signals. The model was able to learn the maps between HS signals and RVSBP. At the same time, RVSBP were estimated without complex features extraction. Three schemes were used to test and verify the Bi-LSTM model. The results indicated that the mean MAE and SD were usually not greater than $5 \mathrm{mmHg}$ in scheme I and scheme II. The model even had good generalization ability across subjects in scheme III. This study suggested an easy operated technique to monitor RVSBP in real-time. It may have potential applications in early detection of right ventricular disease.

Author Contributions: Conceptualization, M.W. and H.T.; methodology, M.W and T.F.; software, M.W.; validation, M.W. and H.T., T.F. and B.G.; formal analysis, H.T.; investigation, M.W. and H.T.; resources, H.T.; data curation, H.T.; writing — original draft preparation, M.W. and H.T.; writing—review and editing, T.F. and B.G.; visualization, M.W. and H.T.; supervision, T.F. and B.G.; project administration, H.T.; funding acquisition, H.T. All authors have read and agreed to the published version of the manuscript.

Funding: This work was supported in part by the National Natural Science Foundation of China under Grant No. 61971089, No. 61471081. National Key R\&D Program of the Ministry of Science and Technology of China, SQ2019YFC200094.

Conflicts of Interest: The authors declare no conflict of interests.

\section{References}

1. Haddad, F.; Hunt, S.A.; Rosenthal, D.N.; Murphy, D.J. Right ventricular function in cardiovascular disease, part I: Anatomy, physiology, aging, and functional assessment of the right ventricle. Circulation 2008, 117, 1436-1448. [CrossRef] [PubMed]

2. Haddad, F.; Doyle, R.; Murphy, D.J.; Hunt, S.A. Right ventricular function in cardiovascular disease, part II: Pathophysiology, clinical importance, and management of right ventricular failure. Circulation 2008, 117, 1717-1731. [CrossRef] [PubMed]

3. Vonk-Noordegraaf, A.; Haddad, F.; Chin, K.M.; Forfia, P.R.; Kawut, S.M.; Lumens, J.; Naeije, R.; Newman, J.; Oudiz, R.J.; Provencher, S.; et al. Right heart adaptation to pulmonary arterial hypertension: Physiology and pathobiology. J. Am. Coll. Cardiol. 2013, 62, D22-D33. [CrossRef] [PubMed]

4. Naeije, R.; Brimioulle, S.; Dewachter, L. Biomechanics of the right ventricle in health and disease (2013 Grover Conference series). Pulm. Circ. 2014, 4, 395-406. [CrossRef] [PubMed]

5. Zornoff, L.A.; Skali, H.; Pfeffer, M.A.; Sutton, M.S.J.; Rouleau, J.L.; Lamas, G.A.; Plappert, T.; Rouleau, J.R.; Moyé, L.A.; Lewis, S.J.; et al. Right ventricular dysfunction and risk of heart failure and mortality after myocardial infarction. J. Am. Coll. Cardiol. 2002, 39, 1450-1455. [CrossRef]

6. Galiè, N.; Humbert, M.; Vachiery, J.L.; Gibbs, S.; Lang, I.; Torbicki, A.; Simonneau, G.; Peacock, A.; Vonk Noordegraaf, A.; Beghetti, M.; et al. 2015 ESC/ERS guidelines for the diagnosis and treatment of pulmonary hypertension: The joint task force for the diagnosis and treatment of pulmonary hypertension of the European Society of Cardiology (ESC) and the European Respiratory Society (ERS): Endorsed by: Association for European Paediatric and Congenital Cardiology (AEPC), International Society for Heart and Lung Transplantation (ISHLT). Eur. Heart J. 2016, 37, 67-119. 
7. Nagel, E.; Stuber, M.; Hess, O. Importance of the right ventricle in valvular heart disease. Eur. Heart J. 1996, 17, 829-836. [CrossRef]

8. Hatano, S.; Strasser, T. Primary Pulmonary Hypertension: Report on a WHO Meeting, GENEVA, 15-17 October 1973; World Health Organization: Geneva, Switzerland, 1975.

9. Kim, N.H.; Delcroix, M.; Jenkins, D.P.; Channick, R.; Dartevelle, P.; Jansa, P.; Lang, I.; Madani, M.M.; Ogino, H.; Pengo, V.; et al. Chronic thromboembolic pulmonary hypertension. J. Am. Coll. Cardiol. 2013, 62, D92-D99. [CrossRef]

10. Barst, R.J.; McGoon, M.; Torbicki, A.; Sitbon, O.; Krowka, M.J.; Olschewski, H.; Gaine, S. Diagnosis and differential assessment of pulmonary arterial hypertension. J. Am. Coll. Cardiol. 2004, 43, S40-S47. [CrossRef]

11. Yock, P.G.; Popp, R.L. Noninvasive estimation of right ventricular systolic pressure by Doppler ultrasound in patients with tricuspid regurgitation. Circulation 1984, 70, 657-662. [CrossRef]

12. Testani, J.M.; Sutton, M.G.S.J.; Wiegers, S.E.; Khera, A.V.; Shannon, R.P.; Kirkpatrick, J.N. Accuracy of noninvasively determined pulmonary artery systolic pressure. Am. J. Cardiol. 2010, 105, 1192-1197. [CrossRef] [PubMed]

13. Chen, D.; Pibarot, P.; Honos, G.; Durand, L.-G. Estimation of pulmonary artery pressure by spectral analysis of the second heart sound. Am. J. Cardiol. 1996, 78, 785-789. [CrossRef]

14. Xu, J.; Durand, L.-G.; Pibarot, P. A new, simple, and accurate method for non-invasive estimation of pulmonary arterial pressure. Heart 2002, 88, 76-80. [CrossRef] [PubMed]

15. Tranulis, C.; Durand, L.-G.; Senhadji, L.; Pibarot, P. Estimation of pulmonary arterial pressure by a neural network analysis using features based on time-frequency representations of the second heart sound. Med Biol. Eng. Comput. 2002, 40, 205-212. [CrossRef]

16. Cherif, L.H.; Debbal, S. Algorithm for the estimation of pulmonary hypertension by the heart sounds using a Hilbert transform. Int. J. Biomed. Eng. Technol. 2016, 20, 356-368. [CrossRef]

17. Nigam, V.; Priemer, R. A dynamic method to estimate the time split between the A2 and P2 components of the S2 heart sound. Int. J. Physiol. Meas. 2006, 27, 553-567. [CrossRef]

18. Dennis, A.; Michaels, A.D.; Arand, P.; Ventura, D. Noninvasive diagnosis of pulmonary hypertension using heart sound analysis. Comput. Biol. Med. 2010, 40, 758-764. [CrossRef]

19. Smith, R.; Ventura, D. A general model for continuous noninvasive pulmonary artery pressure estimation. Comput. Biol. Med. 2013, 43, 904-913. [CrossRef]

20. Yang, Y.; Gillon, S.; Banus, J. Non-invasive Pressure Estimation in Patients with Pulmonary Arterial Hypertension: Data-Driven or Model-Based? In Proceedings of the Statistical Atlases and Computational Models of the Heart, Shenzhen, China, 13 October 2019.

21. Liu, W.; Wang, Z.; Liu, X.; Zeng, N.; Liu, Y.; Alsaadi, F.E. A survey of deep neural network architectures and their applications. Neurocomputing 2017, 234, 11-26. [CrossRef]

22. Luisada, A.A.; Liu, C.K.; Aravanis, C.; Testelli, M.; Morris, J. On the mechanism of production of the heart sounds. Am. Heart J. 1958, 55, 383-399. [CrossRef]

23. Piemme, T.E.; Barnett, G.O.; Dexter, L. Relationship of heart sounds to acceleration of blood flow. Circ. Res. 1966, 18, 303-315. [CrossRef] [PubMed]

24. Pan, J.; Tompkins, W.J. A real-time QRS detection algorithm. IEEE Trans. Biomed. Eng. 1985, 3, $230-236$. [CrossRef] [PubMed]

25. Alpert, B.S.; Quinn, D.E.; Friedman, B.C.; Matsumura, P.M.; Dart, R.A.; Donehoo, R.F. Evaluating the impact of motion artifact on noninvasive blood pressure devices. J. Clin. Hypertens. 2020, 22, 585-589. [CrossRef] [PubMed]

26. Hochreiter, S.; Schmidhuber, J. Long short-term memory. Neural Comput. 1997, 9, 1735-1780. [CrossRef] [PubMed]

27. Graves, A.; Schmidhuber, J. Framewise phoneme classification with bidirectional LSTM and other neural network architectures. Neural Netw. 2005, 18, 602-610. [CrossRef]

28. Stephen, B.; Dalal, P.; Berger, M.; Schweitzer, P.; Hecht, S. Noninvasive estimation of pulmonary artery diastolic pressure in patients with tricuspid regurgitation by Doppler echocardiography. Chest 1999, 116, 73-77. [CrossRef]

29. Tang, H.; Jiang, Y.; Li, T.; Wang, X. Identification of pulmonary hypertension using entropy measure analysis of heart sound signal. Entropy 2018, 20, 389. [CrossRef] 
30. Elgendi, M.; Bobhate, P.; Jain, S.; Guo, L.; Rutledge, J.; Coe, Y.; Zemp, R.; Schuurmans, D.; Adatia, I. The voice of the heart: Vowel-like sound in pulmonary artery hypertension. Diseases 2018, 6, 26. [CrossRef]

31. Elgendi, M.; Bobhate, P.; Jain, S.; Guo, L.; Kumar, S.; Rutledge, J.; Coe, Y.; Zemp, R.; Schuurmans, D.; Adatia, I. The unique heart sound signature of children with pulmonary artery hypertension. Pulm. Circ. 2015, 5, 631-639. [CrossRef]

32. Elgendi, M.; Bobhate, P.; Jain, S.; Rutledge, J.; Coe, J.Y.; Zemp, R.; Schuurmans, D.; Adatia, I. Time-domain analysis of heart sound intensity in children with and without pulmonary artery hypertension: A pilot study using a digital stethoscope. Pulm. Circ. 2014, 4, 685-695. [CrossRef]

33. Elgendi, M.; Bobhate, P.; Jain, S.; Guo, L.; Rutledge, J.; Coe, Y.; Zemp, R.; Schuurmans, D.; Adatia, I. Spectral analysis of the heart sounds in children with and without pulmonary artery hypertension. Int. J. Cardiol. 2014, 173, 92-99. [CrossRef] [PubMed]

34. Kapur, G.; Chen, L.; Xu, Y.; Cashen, K.; Clark, J.; Feng, X.; Wu, S.F. Noninvasive determination of blood pressure by heart sound analysis compared with intra-arterial monitoring in critically III children-A pilot study of a novel approach. Pediatric Crit. Care Med. 2019, 20, 809-816. [CrossRef] [PubMed]

35. Peng, R.C.; Yan, W.R.; Zhang, N.L.; Lin, W.H.; Zhou, X.L.; Zhang, Y.T. Cuffless and continuous blood pressure estimation from the heart sound signals. Sensors 2015, 15, 23653-23666. [CrossRef] [PubMed]

36. Zhang, X.Y.; MacPherson, E.; Zhang, Y.T. Relations between the timing of the second heart sound and aortic blood pressure. IEEE Trans. Biomed. Eng. 2008, 55, 1291-1297. [CrossRef]

37. Zhang, X.Y.; Zhang, Y.T. Model-based analysis of effects of systolic blood pressure on frequency characteristics of the second heart sound. In Proceedings of the 2006 International Conference of the IEEE Engineering in Medicine and Biology Society, New York, NY, USA, 30 August-3 September 2006; pp. 2888-2891.

(C) 2020 by the authors. Licensee MDPI, Basel, Switzerland. This article is an open access article distributed under the terms and conditions of the Creative Commons Attribution (CC BY) license (http://creativecommons.org/licenses/by/4.0/). 